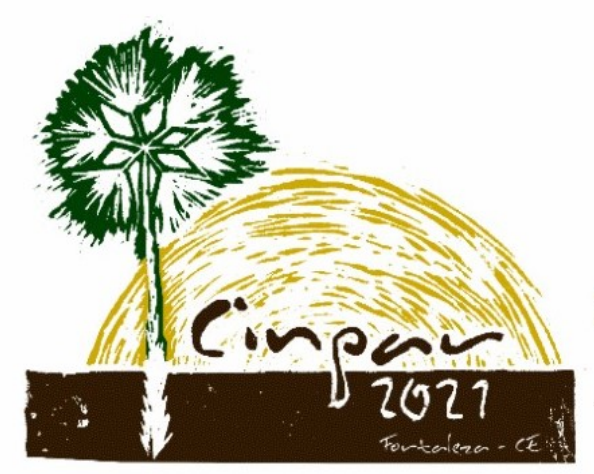

XVII Congresso Internacional sobre Patologia e Reabilitação das Construções

XVII Congreso Internacional sobre Patología y Rehabilitación de las Construcciones

XVII International Conference on Pathology and Constructions Rehabilitation

FORTALEZA (Brasil), 3 a 5 de junho de 2021 https://doi.org/10.4322/CINPAR.2021.081

\title{
Sistema computacional baseado em reconhecimento de padrões para classificação de concretos
}

\section{Computer system based on pattern recognition to classify concretes}

\author{
Alessandra I. P. dos SANTOS ${ }^{1}$, Eduarda S. GAVIÃO ${ }^{2}$, Sara R. B. MARIN ${ }^{3}$, Carlos E. T. BALESTRA ${ }^{4}$, Fabio A. \\ SPANHOL ${ }^{4}$, Jefferson G. MARTINS ${ }^{5}$

\footnotetext{
${ }^{1}$ Universidade Tecnológica Federal do Paraná (UTFPR), Toledo, Brasil, aleiolandap@hotmail.com ${ }^{2}$ Universidade Tecnológica Federal do Paraná (UTFPR), Toledo, Brasil, eduardagaviao@hotmail.com

${ }^{3}$ Universidade Tecnológica Federal do Paraná (UTFPR), Toledo, Brasil, sarabocato@hotmail.com

${ }^{4}$ Universidade Tecnológica Federal do Paraná (UTFPR), Toledo, Brasil, carlosbalestra@utfpr.edu.br

${ }^{5}$ Universidade Tecnológica Federal do Paraná (UTFPR), Toledo, Brasil, faspanhol@utfpr.edu.br

${ }^{6}$ Universidade Tecnológica Federal do Paraná (UTFPR), Toledo, Brasil, martins@utfpr.edu.br
}

\begin{abstract}
The proportion of materials used in concrete composition has great importance in order to build a structure and public security, since materials have influence on concrete properties, in fresh and hardened states. The identification of concrete traces is usually carried by human experts with high levels of experience, but their results are influenced by physical and subjective factors. This paper presents a proposal based on pattern recognition to identify different concrete traces through out images and their texture features. The achieved results demonstrated the possibility of identifying concrete traces through application of pattern recognition techniques to images. The best achieved rate was $87.1 \%$ of accuracy using the SURF descriptor.
\end{abstract}

Keywords: Construction. Public security. Machine learning. Textural descriptors.

Resumo: A proporção dos materiais que compõem o concreto tem grande importância para a construção de uma estrutura e segurança de seus usuários, pois os materiais influenciam nas propriedades do concreto, tanto em estado fresco como em estado endurecido. A identificação dos traços de concreto normalmente é realizada por especialistas humanos com altos níveis de experiência, sendo os resultados possivelmente influenciados por fatores físicos e subjetivos. Este artigo apresenta uma proposta baseada em reconhecimento de padrões para identificar diferentes traços de concreto por meio de imagens e suas características texturais. Os resultados alcançados demonstraram a possibilidade de identificação de traços de concreto por meio da aplicação de técnicas de reconhecimento de padrões a imagens. A melhor taxa alcançada foi $87,1 \%$ de acurácia com o descritor SURF.

Palavras-chave: Construção civil. Segurança pública. Aprendizagem de máquinas. Descritores texturais. 


\section{Introdução}

A composição do concreto, também chamada traço, é caracterizada por seus componentes e as respectivas quantidades. Tal definição é uma característica extremamente importante em qualquer obra e muda de acordo com a finalidade de aplicação: lajes, contrapisos, muros, fundações, calçadas, vigas, etc. Além disso, a proporção e a origem dos materiais exercem influência nas características finais do traço, dentre as quais tem-se resistência, durabilidade e trabalhabilidade. Dentre os materiais comumente utilizados, tem-se cimento, areia, brita e água, sendo que concretos com características especiais ainda incluem aditivos, isopor, pigmentos, fibras, dentre outros (ABNT NBR 12655, 2015; MALTA, 2012; MONTEIRO, 2010).

A função dos agregados é aumentar o volume final e torná-lo mais econômico. Estes têm influências da formação geológica local, pois são extraídos de jazidas próximas ao local de dosagem e edificação. Conhecer suas características individuais, e também de sua combinação, é essencial para garantir certas características fundamentais ao concreto e a segurança da estrutura. Além disso, materiais com diferentes granularidades podem ser combinados dependendo da finalidade e produzem diferentes características texturais (ABNT NBR 12655, 2015; BAUER, 1994; MALTA, 2012; METHA et al., 2008; MONTEIRO, 2010).

$A$ água deve ser suficiente para envolver os grãos e promover a hidratação do cimento, além de atender os requisitos da ABNT NBR 15900-1:2009. Sua função é ativar a reação química que transforma o cimento em uma pasta aglomerante que criará um bloco único com a areia e a brita (agregados). Se em excesso, ter-seá uma pasta mais porosa e melhores níveis de trabalhabilidade. Porém, também haverá piores níveis de resistência e aderência entre a pasta e o agregado devido à exsudação. Para a definição de tal proporção é necessário identificar o teor de umidade dos agregados, pois estes podem transportar diferentes quantidades de água para o concreto e ocasionar o decréscimo de sua resistência mecânica (ABNT NBR 15900-1, 2009; ABNT NBR 12655, 2015; MALTA, 2012; MONTEIRO, 2010).

Além dos custos envolvidos devido à degradação destas estruturas, o risco em termos de colapsos estruturais merece atenção. Nos últimos anos, têm sido recorrentes os casos de colapsos de estruturas, dentre os quais tem-se o colapso parcial de um viaduto em Brasília no ano de 2018 (VIADUTO, 2018), a interdição do acesso à Rodovia Presidente Dutra em São Paulo no ano de 2019 (PREFEITURA, 2019) e o desabamento de prédios na comunidade de Muzema, na Zona Oeste do Rio, em 2019 (MAIA et al., 2019). Portanto, estudos pertinentes que auxiliem a fiscalização e a prevenção destes acontecimentos são necessários com vistas a garantir estruturas mais seguras e duráveis e manutenções preventivas.

Outra importante característica deste cenário compreende o fato de que as inspeções para avaliar a composição do concreto, e também se este mantém suas propriedades, são realizadas visualmente por especialistas humanos. Tais inspeções demandam altos níveis de experiência por ser um fator decisivo para uma correta avaliação. Dentre as possíveis ferramentas a serem empregadas, tem-se filmadoras, máquinas fotográficas, lupas e binóculos (ABNT NBR 6118, 2004; SCHVAICKARDT et al., 2018).

Diante do exposto, este artigo apresenta uma alternativa para auxiliar as inspeções realizadas por especialistas humanos na identificação dos constituintes do concreto de uma maneira qualitativa por meio de imagens e reconhecimento de padrões. A Figura 1 ilustra o problema abordado no projeto e seu grau de dificuldade. Suas imagens foram obtidas de cortes transversais de dois diferentes traços (cimento : areia : brita : água), com as proporções (1: $1,41: 2,01: 0,49)$ e $(1: 1,49: 2,60: 0,49)$. Neste exemplo, pode-se nitidamente observar a variação na quantidade de britas entre os concretos. A proposta apresentada propicia vantagens relativas à rapidez e precisão quando comparado à avaliação realizada manualmente. A redução de fatores físicos e subjetivos inerentes ao ser humano também reduz o número de etapas executadas manualmente e sua influência nos resultados finais. Tal influência compreende questões como subjetividade dos especialistas humanos e características do processo (repetitivo, monótono e demorado), além de requerer alto grau de concentração. Este conjunto de exigências sobrecarrega o profissional que realiza a inspeção e o leva a possíveis distrações e baixas taxas de acerto (CONNERS et al., 1997; PHAM et al., 1997; RADOVAN et al., 2001). 


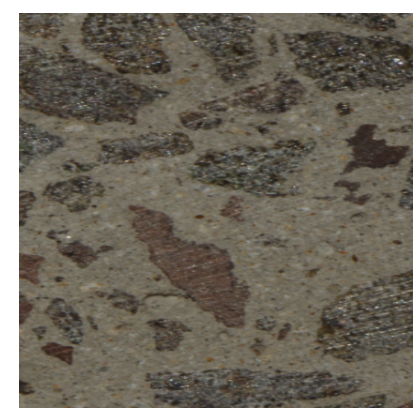

(a)

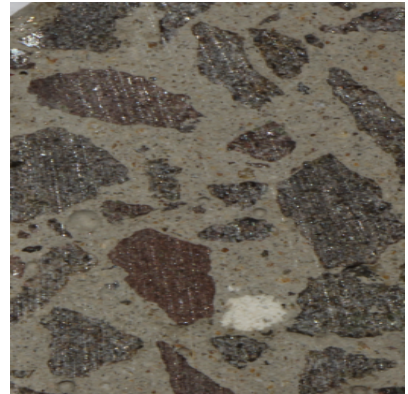

(b)

Figura 1 - Composições de concreto (cimento : areia : brita : água): (a) $1: 1,41: 2,01: 0,49$; (b) $1: 1,49: 2,60: 0,49$

\section{Materiais e métodos}

Sistemas computacionais com foco em reconhecimento de padrões contemplam as seguintes etapas: aquisição, pré-processamento, segmentação, extração de características e classificação. Cada etapa constitui um diferente contexto, possui diferentes níveis de complexidade e envolve conhecimentos específicos e próprios, além daqueles inerentes ao domínio da aplicação.

$\mathrm{Na}$ primeira etapa foi obtida a base de dados, ou seja, o conjunto de imagens. Inicialmente foram preparados os corpos de prova de concreto sob a forma cilíndrica e com medidas de $10 \mathrm{~cm}$ de diâmetro e $20 \mathrm{~cm}$ de altura. Foram utilizadas dois corpos de prova de concreto para cada um dos diferentes traços analisados. Tal escolha é consequência do fato (e se adequa a ele) de que dois corpos de prova são construídos com amostras de cada carga de concreto utilizada nas construções.

Os traços utilizados para a construção dos corpos de prova consideraram a proporção $1: 1,41: 2,01: 0,49$ utilizando cimento do tipo Portland CPII, areia média, brita zero (dimensão máxima característica igual a 9,5 $\mathrm{mm}$ ) e água potável disponibilizada pela companhia de saneamento. Para avaliar a variação da granularidade do agregado brita, foi utilizada uma variação do traço padrão com a troca da brita zero pela brita um (dimensão máxima característica igual a $19 \mathrm{~mm}$ ), gerando o traço $1: 1,49: 2,60: 0,49$. Nesta variação de traço, tem-se um uso maior dos agregados (areia e brita) devido a um maior volume e superfície de cada unidade do agregado brita, sendo necessária menor quantidade proporcional de cimento para a construção de peças com um mesmo volume final, tal como ilustram as imagens obtidas de cortes transversais apresentados na Figura 1.

Na sequência, foram realizados cortes transversais a cerca de $5 \mathrm{~mm}$ da base e outro $5 \mathrm{~mm}$ do topo do corpo de prova. Tais cortes estão ilustrados na Figura 2(a) e o objetivo de ambos os cortes é eliminar a camada superficial e expor a caracterização da composição real do concreto, tal como apresenta a Figura 2(b). A partir disto, foram realizados outros oito cortes transversais em cada corpo de prova de concreto. Considerando que as lâminas de corte têm aproximadamente $2 \mathrm{~mm}$, sendo obtido um total de nove amostras distintas para cada corpo de prova. Considerando as duas faces de cada amostra, chegou-se a um total de 18 imagens para cada corpo de prova. Ao todo, foram utilizadas 72 imagens para construção dos modelos de reconhecimento dos traços de concreto.

O pré-processamento aplica operações para realçar as características importantes no processo de diferenciação das classes existentes no problema. Nesta etapa, basicamente foi utilizada a biblioteca OpenCV para converter a base toda em escala de cinza, tal como ilustra as imagens da Figura 2(b-c).

A segmentação compreende a próxima etapa e busca separar a região de interesse da imagem original e da qual depende o sucesso de um modelo de identificação ou de classificação. Para isso, uma imagem é "fragmentada" em múltiplas regiões, com o objetivo de simplificar ou mudar sua representação e facilitar sua análise. Nesta etapa, buscou-se por uma imagem que estivesse toda formada pela área de interesse, Figura 2(f). As imagens da Figura 2(c-d) representam a aplicação da técnica de limiarização para destacar o objeto na imagem. Na sequência, aplicou-se o algoritmo de detecção de bordas Canny (BUENO, 2020) para 
ressaltar os contornos das bordas dos objetos e a função findContours da biblioteca OpenCV para identificar os objetos. Esta função retorna os contornos de todos os possíveis objetos presentes na imagem, seja do próprio traço de concreto, das britas ou de qualquer outra região com contraste em relação a seu entorno. A partir da identificação dos possíveis objetos, os pontos pertencentes ao maior contorno com a definição do traço de concreto foram selecionados. Após o cálculo do raio da circunferência, definiu-se e salvou-se o retângulo circunscrito na mesma, Figura 2(e), culminando na Figura 2(f).

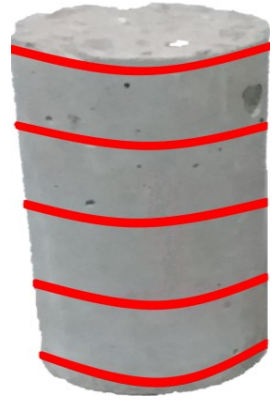

(a)

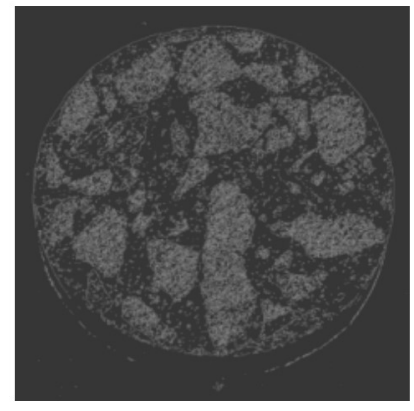

(d)

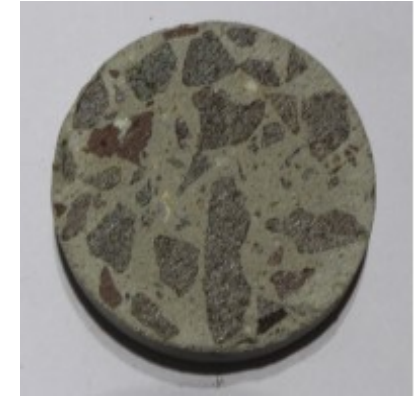

(b)

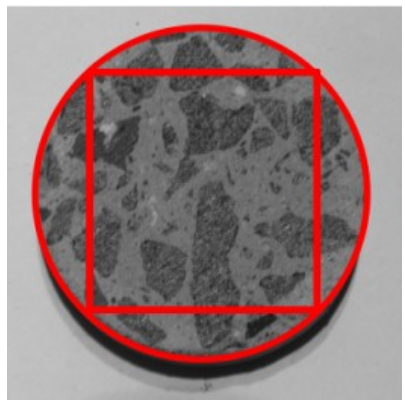

(e)

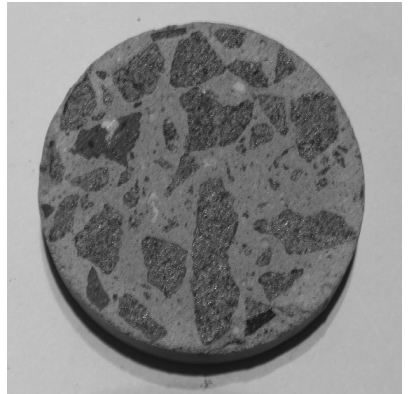

(c)

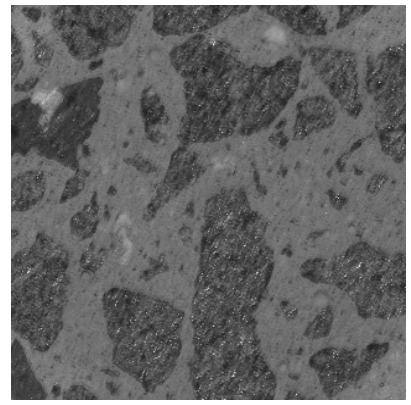

( f)

Figura 2 - Ilustração do processo de reconhecimento de padrões: ( a ) corpo de prova de concreto; ( b ) corte do corpo de prova; ( c ) pré-processamento com conversão para níveis de cinza; ( d-e ) segmentação

A Extração de Características permite identificar uma abstração (descritor ou conjunto de características) adequada para representar e descrever as áreas de interesse, a qual é constituída por um vetor numérico de medidas. Foram utilizadas implementações disponíveis no software MatLab 2016 para a obtenção dos descritores. Foi empregado o amplamente conhecido Padrão Binário Local (LBP - Local Binary Pattern), por meio de suas variantes LBP u2 $_{2}$ e LBP riuz (MÄEMPÄ et al., 2000; OJALA et al., 1996). Além destes, outros três descritores baseados em pontos de atenção foram considerados, Transformação de Características Invariantes à Escala (Scale Invariant Feature Transform - SIFT) (LOWE, 1999, 2004; VEDALDI et al., 2008), Características Robustas Aceleradas (Speed-Up Robust Feature - SURF) (BAY et al., 2006, 2008) e Regiões Extremas Maximamente Estáveis (Maximally Stable Extremal Regions - MSER) (MATAS et al., 2002).

Pontos de atenção são empregados na identificação de objetos durante seu rastreamento em vídeos, principalmente no monitoramento de ambientes como aeroportos. As características extraídas dos pontos de atenção detectados possuem alto potencial devido aos padrões texturais decorrentes das composições dos traços de concreto (Figura 1). Devido à variação do número de regiões e pontos identificados em cada imagem, optou-se por utilizar momentos estatísticos para padronizar sua representação. Foram calculadas média, variância, obliquidade e curtose para cada coluna dos vetores gerados pelos descritores. Ao final, obteve-se quatro vetores de características para cada imagem, sendo um para cada momento estatístico anteriormente definido. Para SIFT foram gerados vetores com 128 elementos para cada ponto de atenção identificado nas imagens. Já para SURF e MSER foram consideradas as versões com 64 e 128 elementos 
para cada ponto de atenção identificado. Esta abordagem é comumente utilizada com filtros de Gabor (YANG et al., 2008; ZHU et al., 2008). Maiores detalhes quanto à metodologia utilizada para a estração e a variação de parâmetros podem ser obtidos em Martins (2014).

A classificação utiliza as representações anteriores para diferenciar os objetos nas imagens, atribuindo-lhes identificadores e significados de acordo com suas características e descritores (PEDRINI et al., 2008). Na etapa de classificação foi utilizado o algoritmo Máquina de Vetores de Suporte (Support Vector Machine SVM), por meio da implementação LibSVM 3.2 (CHANG et al., 2011). Dentre as possibilidades disponibilizadas pela biblioteca, os melhores resultados foram obtidos com a função de base radial Gaussiana e uma busca gulosa para encontrar os melhores parâmetros $C$ e $\gamma$. A normalização dos dados considerou a escala linear de cada atributo dos vetores de características no intervalo $[-1,+1]$.

Diferentes avaliações foram consideradas a partir dos vetores de características com os momentos estatísticos anteriormente descritos, além de uma avaliação utilizando apenas número de pontos de atenção identificados em cada imagem como característica. Para a definição dos conjuntos de treinamento e teste, dividiu-se a base de imagens em três partes (folds), as quais mantinham a representatividade das classes pertencentes ao problema em questão. Foi empregada validação cruzada com três execuções, sendo que em que execução, uma parte era usada como conjunto de teste e as outras duas eram utilizadas como conjunto de treinamento. Os resultados finais são expressos pela acurácia média (acertos do classificador) e desvio padrão (dp) das taxas de classificações corretas das três execuções.

\section{Resultados}

Para cada etapa do processo de reconhecimento de padrões foram testados diferentes parâmetros, muitos dos quais já identificados anteriormente na seção de materiais e métodos. Os melhores resultados são apresentados nos Quadros 1 à 4. As melhores taxas de reconhecimento obtidas com o descritor LBP foram $79.2 \%(\mathrm{dp}=2.4)$ com a versão $\mathrm{LBP}_{\text {riuz }}$ e sua invariância à rotação.

Quadro 1 - Taxas de classificações corretas para o Descritor LBP

\begin{tabular}{|c|c|c|c|c|}
\hline Versão do LBP & $\mathbf{P}$ & $\mathbf{R}$ & Acurácia (\%) & $\mathbf{d p}$ \\
\hline \multirow{3}{*}{ riu2 } & 8 & 1 & 69.2 & 4.7 \\
\cline { 2 - 5 } & 8 & 2 & 73.3 & 6.2 \\
\cline { 2 - 5 } & 16 & 2 & 79.2 & 2.4 \\
\cline { 2 - 5 } & 24 & 3 & 72.5 & 8.2 \\
\hline \multirow{4}{*}{ u2 } & 8 & 1 & 35.8 & 20.9 \\
\cline { 2 - 5 } & 8 & 2 & 67.5 & 12.4 \\
\cline { 2 - 5 } & 16 & 2 & 50.8 & 26.3 \\
\cline { 2 - 5 } & 24 & 3 & 52.5 & 22.5 \\
\hline
\end{tabular}

Quadro 2 - Taxas de classificações corretas para o Descritor SIFT

\begin{tabular}{|c|c|c|}
\hline Medida & Acurácia (\%) & dp \\
\hline \# Pontos & 56.4 & 3.2 \\
\hline Média & 81.3 & 2.8 \\
\hline Variância & $\mathbf{8 4 . 8}$ & 2.9 \\
\hline Obliquidade & 81.4 & 7.9 \\
\hline Curtose & 82.5 & 5.3 \\
\hline
\end{tabular}


Quadro 3 - Taxas de classificações corretas para o Descritor SURF

\begin{tabular}{|c|c|c|c|}
\hline Versão do SURF & Medida & Acurácia (\%) & dp \\
\hline \multirow{4}{*}{64} & \# Pontos & 57.6 & 1.4 \\
\cline { 2 - 4 } & Média & 82.2 & 3.5 \\
\cline { 2 - 4 } & Variância & $\mathbf{8 7 . 1}$ & 3.1 \\
\cline { 2 - 4 } & Obliquidade & 69.3 & 2.4 \\
\cline { 2 - 4 } & Curtose & 74.1 & 7.0 \\
\hline \multirow{4}{*}{128} & \# Pontos & 57.6 & 1.4 \\
\cline { 2 - 4 } & Média & 78.9 & 2.3 \\
\cline { 2 - 4 } & Variância & $\mathbf{8 7 . 1}$ & 3.1 \\
\cline { 2 - 4 } & Obliquidade & 72.9 & 2.3 \\
\cline { 2 - 4 } & Curtose & 74.0 & 6.2 \\
\hline
\end{tabular}

Quadro 4 - Taxas de classificações corretas para o Descritor MSER-SURF

\begin{tabular}{|c|c|c|c|}
\hline Versão do SURF & Medida & Acurácia (\%) & dp \\
\hline \multirow{4}{*}{64} & \# Pontos & 62.5 & 5.5 \\
\cline { 2 - 4 } & Média & 63.4 & 6.4 \\
\cline { 2 - 4 } & Variância & 57.8 & 9.4 \\
\cline { 2 - 4 } & Obliquidade & 61.2 & 1.9 \\
\cline { 2 - 4 } & Curtose & $\mathbf{6 3 . 5}$ & 4.2 \\
\hline \multirow{4}{*}{128} & \# Pontos & 62.5 & 5.5 \\
\cline { 2 - 4 } & Média & 61.1 & 3.1 \\
\cline { 2 - 4 } & Variância & $\mathbf{7 4 . 2}$ & 3.8 \\
\cline { 2 - 4 } & Obliquidade & 56.5 & 1.9 \\
\cline { 2 - 4 } & Curtose & 54.2 & 6.7 \\
\hline
\end{tabular}

Para SIFT, os melhores resultados alcançados foram $84.8 \%(\mathrm{dp}=2.9$ ) por meio dos vetores de características definidos pelo cálculo do momento estatístico variância (Quadro 2). No Quadro 3 tem-se os resultados obtidos com o descritor SURF, com o qual proveu taxas de reconhecimento de $87.1 \%(\mathrm{dp}=3.1$ ) com vetores de características baseados no momento estatístico variância para ambas as versões. Utilizando a combinação SURF-MSER (Quadro 4) atingiu-se $74.2 \%(\mathrm{dp}=3.8$ ) para a versão com 128 atributos e o momento estatístico variância.

O problema em questão envolve segurança e vidas humanas, além dos altos valores monetários empregados na construção e manutenção das edificações. Os resultados obtidos se mostraram importantes, principalmente com os bons resultados alcançados nos experimentos preliminares apresentados. Além do exposto, destaca-se o descritor SURF com os melhores resultados utilizando apenas vetores de características com 64 atributos. 


\section{Conclusões}

Este trabalho abordou ao problema de reconhecimento de traços de concreto por meio de imagens. Foram avaliados classificadores construídos a partir do amplamente conhecido descritor LPB, por meio de suas variantes $\mathrm{LBP}_{\mathrm{uz}}$ e $\mathrm{LBP}_{\text {riu2}}$, além dos descritores SIFT, SURF e MSER-SURF, baseados em pontos de atenção. Os resultados alcançados são bastante promissores para problema abordado, sendo que a melhor taxa de reconhecimento foi $87.1 \%$ ( $\mathrm{dp}=3.1$ ) usando apenas 64 atributos extraídos para o descritor SURF. Tais resultados corroboram com a proposta de identificação de traços de concreto por meio da aplicação de técnicas de reconhecimento de padrões e imagens.

Os trabalhos futuros compreenderão a ampliação da base de imagens, com maior variedade de traços e imagens. Ao mesmo tempo, vislumbra-se a continuidade dos trabalhos com a avaliação de novas alternativas para descritores, classificadores, estratégias de validação e também a seleção e combinação de modelos, com o intuito de melhorar ainda mais os resultados. Possíveis alternativas foram apresentadas por Martins et al. (2012), Martins (2014), Martins et al. (2015) e Spanhol (2018).

\section{Referências Bibliográficas}

ASSOCIAÇÃO BRASILEIRA DE NORMAS TÉCNICAS - ABNT NBR 6118 (2004). ABNT NBR 6118: Projeto de estruturas de concreto - Procedimento. Rio de Janeiro, 2004.

ASSOCIAÇÃO BRASILEIRA DE NORMAS TÉCNICAS - ABNT NBR 15900-1 (2009). ABNT NBR 15900-1: Água para amassamento do concreto Parte 1: Requisitos. Rio de Janeiro, 2009.

ASSOCIAÇÃO BRASILEIRA DE NORMAS TÉCNICAS - ABNT NBR 12655 (2015). ABNT NBR 12655: Concreto de cimento Portland - Preparo, controle, recebimento e aceitação - Procedimento. Rio de Janeiro, 2015.

BAUER, L. A. F. (1994). Materiais de Construção. 5.ed. v. 1. Rio de Janeiro: LTC Editora S.A.

BAY, H.; TUYTELAARS, T.; e VAN GOOL, L. (2006). SURF: Speeded up robust features. ECCV, 404-417.

BAY, H.; ESS, A.; TUYTELAARS, T.; e VAN GOOL, L. (2008) Speeded-up robust features (SURF). Compute Vision. Image Understanding, 110 (3), 346-359.

BUENO, M. L. (2020). Deteç̧ão de Bordas através de Algoritmo Canny. Disponível em: http://www.inf.ufsc.br/ aldo.vw/visao/2000/Bordas/index.htm. Acesso em: 20 jan. 2020.

CHANG, C.-C.; LIN, C.-J. (2011). LIBSVM: a library for support vector machines. ACM Transactions on Intelligent Systems and Technology, 2(3), 1-27. Disponível em: http://www.csie.ntu.edu.tw/ cjlin/libsvm/. Acesso em: 20 de jun. de 2020.

CONNERS, R. W.; KLINE, D. E.; ARAMAN, P. A.; DRAYER, T. H. (1997) Machine vision technology for the forest products industry. Computer, 30 (7), 43-48.

LÓPES, G. A. P. (2011). Aforapro: reconhecimento de objetos invariante sob transformações afins. Dissertação de Mestrado, Escola Politécnica da Universidade de São Paulo, São Paulo.

LOWE, D.G. (1999). Object recognition from local scale-invariant features. In International Conference on Computer Vision, 2, 1150-, Washington, DC.

LOWE, D.G. (2004). Distinctive image features from scale-invariant keypoints. In Int. Journal of Computer Vision, 60 (2), 91-110.

MÄENPÄÄ, T.; OJALA, T. PIETIKÄINEN, M.; SORIANO, M. (2000). Robust texture classification by subsets of local binary patterns. 15th International Conference on Pattern Recognition, 947-950.

MAIA, D. e GARCIA, D. (2019). Dois prédios desabam na zona oeste do Rio de Janeiro. Folha de São Paulo, São Paulo - SP, 12 de abr. de 2019. Disponível em: https://www1.folha.uol.com.br/cotidiano/2019/04/dois-predios-desabam-na-zona-oeste-do-rio-dejaneiro.shtml\#: :text=\%E2\%80\%8BO\%20desabamento\%20de\%20dois,adolescente\%20est\%C3\%A30\%20 entre\%20as\%20v\%C3\%ADtimas.\&text=\%E2\%80\%8BOs\%20pr\%C3\%A9dios\%20ca\%C3\%ADram\%20por\%2 Ovolta\%20das\%206h30. Acesso em: 20 de jun. de 2019. 
MALTA, J. O. (2012). Dosagem de concretos produzidos com agregado miúdo reciclado de resíduo de construção e demolição. Dissertação de Mestrado em Engenharia Ambiental Urbana. Escola Politécnica (EPUFBA). Universidade Federal da Bahia. Salvador.

MARTINS, J.G. (2014). Identificação de Espécies Florestais utilizando Seleção Dinâmica de Classificadores no Espaço de Dissimilaridade. Tese de Doutoramento. Programa de Pós-Graduação em Informática. Universidade Federal do Paraná, Curitiba-PR.

MARTINS, J.G.; OLIVEIRA, L.E.S.; SABOURIN, R. (2012). Combining textural descriptors for forest species recognition. IECON 2012 - 38th Annual Conference on IEEE Industrial Electronics Society, 1483-1488.

MARTINS, J.G.; OLIVEIRA, L.E.S.; BRITTO, A. S.; SABOURIN, R. (2015). Forest species recognition based on dynamic classifier selection and dissimilarity feature vector representation. Machine Vision and Applications, 26 (2), 279-293.

MATAS, J.; CHUM, O.; MARTIN, U.; e PAJDLA, T. (2002). Robust wide baseline stereo from maximally stable extremal regions. British Machine Vision Conference, p. 384-393.

MEHTA, P.K.; MONTEIRO, P. (2008). Concreto: microestrutura, propriedades e materiais. 3.ed. São Paulo: IBRACON.

MONTEIRO, A. C. N. (2010). Concreto Poroso: dosagem e desempenho. Monografia de Graduação em Engenharia Civil. Universidade Federal de Goiás. Goiania-GO.

OJALA, T.; PIETIKÄINEN, M. ; HARWOOD, D. (1996). A comparative study of texture measures with classification based on featured distributions. Pattern Recognition, 29(1), 51-59.

PEDRINI, H.; SCHWARTZ, W.R. (2008). Análise de Imagens Digitais: princípios, algoritmos e aplicações. São Paulo: Thomson Learning.

PHAM, D. T.; ALCOCK, R. J. (1997). Automated visual inspection of birch wood boards. IEE Colloquium on Artificial Intelligence in Manufacturing, 1-4.

PREFEITURA de SP interdita ponte que leva à Dutra pela Marginal Tietê. G1 SP, São Paulo - SP, 23 de jan. de 2019. Disponível em: https://g1.globo.com/sp/sao-paulo/noticia/2019/01/23/prefeitura-de-spinterdita-ponte-que-leva-a-dutra-pela-marginal-tiete.ghtml. Acesso em: 20 de jun. de 2019.

RADOVAN, S.; GEORGE, P.; PANAGIOTIS, M.; MANOS, G.; ROBERT, A.; IGOR, D. (2001). An approach for automated inspection of wood boards. International Conference on Image Processing, 1, 798-801.

SCHVAICKARDT, C. M.; MATTOS, J. R. G. (2018). Estudo de traço para peças prémoldadas de concreto para pavimentos intertravados. Revista Nacional de Gerenciamento de Cidades, 6 (39).

SPANHOL, F. A. (2018). Automatic Breast Cancer Classification From Histopathological Images: A Hybrid Approach. Tese de Doutoramento. Programa de Pós-Graduação em Informática. Universidade Federal do Paraná, Curitiba-PR.

VEDALDI, A.; FULKERSON, B. (2008). VLFeat: An Open and Portable Library of Computer Vision Algorithms. URL: http://www.vlfeat.org/.

VIADUTO da Galeria dos Estados desaba e abre cratera no Eixão Sul. Correio Braziliense, Brasília - DF, 06 de fev. $\quad$ de 2018.2 Disponível em: https://www.correiobraziliense.com.br/app/noticia/cidades/2018/02/06/interna_cidadesdf,658118/par te-do-viaduto-da-galeria-dos-estados-desaba-no-centro-de-brasilia.shtml. Acesso em: 20 de jun. de 2019.

YANG, Y.; e NEWSAM, S. (2008). Comparing SIFT descriptors and Gabor texture features for classification of remote sensed imagery. 15th IEEE International Conference on Image Processing, 1852-1855.

ZHU, J.; HOI, S. C.H.; LYU, M. R.; e YAN, S. (2008). Near-duplicate keyframe retrieval by nonrigid image matching. In 16th ACM international conference on Multimedia, 41-50, New York, NY. 